\title{
Modelling of Supercapacitors: Factors Influencing Performance
}

\author{
Martin Kroupa, ${ }^{a}$ Gregory J. Offer, ${ }^{\text {b,z }}$ and Juraj Kosek ${ }^{\mathrm{a}, \mathrm{z}}$ \\ ${ }^{a}$ Department of Chemical Engineering, University of Chemistry and Technology Prague, 16628 Prague 6, \\ Czech Republic \\ ${ }^{b}$ Department of Mechanical Engineering, Imperial College London, London SW7 2AZ, United Kingdom
}

\begin{abstract}
The utilizable capacitance of Electrochemical Double Layer Capacitors (EDLCs) is a function of the frequency at which they are operated and this is strongly dependent on the construction and physical parameters of the device. We simulate the dynamic behavior of an EDLC using a spatially resolved model based on the porous electrode theory. The model of Verbrugge and Liu (J. Electrochem. Soc. 152, D79 (2005)) was extended with a dimension describing the transport into the carbon particle pores. Our results show a large influence of the electrode thickness $\left(L_{\mathrm{e}}\right)$, separator thickness $\left(L_{\mathrm{s}}\right)$ and electrolyte conductivity $(\kappa)$ on the performance of EDLCs. In agreement with experimental data, the time constant was an increasing function of $L_{\mathrm{e}}$ and $L_{\mathrm{s}}$ and a decreasing function of $\kappa$. The main limitation was found to be on the scale of the whole cell, while transport into the particles became a limiting factor only if the particle size was unrealistically large. The results were generalized into a simplified relation allowing for a quick evaluation of performance for the design of new devices. This work provides an insight into the performance limitation of EDLCs and identifies the critical parameters to consider for both systems engineers and material scientists.

(c) The Author(s) 2016. Published by ECS. This is an open access article distributed under the terms of the Creative Commons Attribution Non-Commercial No Derivatives 4.0 License (CC BY-NC-ND, http://creativecommons.org/licenses/by-nc-nd/4.0/), which permits non-commercial reuse, distribution, and reproduction in any medium, provided the original work is not changed in any way and is properly cited. For permission for commercial reuse, please email: oa @electrochem.org. [DOI: 10.1149/2.0081613jes] All rights reserved.

(cc) BY-NC-ND
\end{abstract}

Manuscript submitted June 24, 2016; revised manuscript received August 31, 2016. Published September 12, 2016.

Electrochemical Double Layer Capacitors (EDLCs) store energy by the adsorption of ions from an electrolyte (resulting in its capacitive deionization), storing the ions in the electrochemical double layer of a charged electrode with a very large surface area. The high charge that can be stored gives rise to the name supercapacitors or ultracapacitors. The main advantages of EDLCs when compared to batteries are their ability to quickly release the stored energy, their high efficiency and their long cycle life. The amount of energy that can be stored is, however, not unlimited. The useable capacitance is dependent on the time scale of their operation and this causes the capacitance of EDLCs to be a function of frequency. As typical application of EDLCs requires them to supply or accept pulses of energy at frequencies ranging from $10 \mathrm{~Hz}$ to $0.1 \mathrm{~Hz},{ }^{1}$ it is important that the full capacity can be utilized in this range. In order to improve the high-frequency behavior, it is necessary to understand the factors causing the limitation and to identify the critical ones.

The key to the high capacitance of EDLCs is the high specific surface area (SSA) of their electrodes. This is achieved by using highly porous activated graphitic carbon materials. ${ }^{2}$ According to their size, the pores can be classified as micropores $(<2 \mathrm{~nm})$, mesopores $(2-50 \mathrm{~nm})$ or macropores $(>50 \mathrm{~nm}){ }^{2}$ Pores of different sizes are connected in a highly complex hierarchical structure depending on the shape of carbon particles. A typical supercapacitor cell consists of two carbon electrodes with the porous separator in between and current collectors closing the cell from both sides.

Experimentally, the frequency-dependent capacitance is commonly measured using either Cyclic Voltammetry (CV), Electrochemical Impedance Spectroscopy (EIS) or Galvanostatic Cycling (GC). ${ }^{3,4}$ Yoon et al. (2005) and Pandolfo et al. (2010) investigated the effect of the electrode thickness $L_{\mathrm{e}}$ on the capacitance clearly showing the capacitance to decay at smaller frequencies for thicker electrodes. ${ }^{5-7} \mathrm{On}$ the other hand, increasing conductivity was found by Lust et al. (2004) to increase the capacitance available at high frequencies. ${ }^{8}$ The separator does not contribute to the capacitance and acts as an additional ionic resistance in the system. ${ }^{9,10}$

Models on the scale of the whole cell are generally based either on the equivalent-circuit (EC) approach or porous electrode theory, and the existing models were recently reviewed by Ike (2015) and Pilon et al. (2015).11,12 Verbrugge and Liu (2005) used a model based on the porous electrode theory to calculate the Ragone plot, which relates the energy density and power density. ${ }^{13}$ This was however done only

zE-mail: gregory.offer@imperial.ac.uk; Juraj.Kosek@vscht.cz for one set of parameters. Using a similar formulation of the model, Allu et al. (2014) performed parametric studies investigating the effect of transport properties in the following setup: constant current charge followed constant voltage discharge. ${ }^{14}$ In a study focused on the usage of the model for control purposes, Drummond et al. (2015) used the Verbrugge model to compute the dependence of the complex capacitance on frequency by means of EIS. Srinivasan et al. (1999) presented an analytical solution of a simplified EDLC model under the operating condition of EIS, capturing the effect of electrode thickness in the capacitance vs. frequency plot. ${ }^{15}$ On the other hand, the classical de Levie equivalent-circuit model on the scale of one mesopore can be analytically solved for different operating conditions, such as step voltage change or oscillatory voltage or current input. ${ }^{16}$ Using the transmission line model, Eikerling et al. (2005) modelled the hierarchical structure of porous electrodes and showed the effect of carbon particle size in the Electrochemical Impedance Spectroscopy simulations. ${ }^{17}$

The main limitation of these equivalent circuit approaches is that they do not capture the whole physical nature of the system. Although they can successfully reproduce the electrical response, the effect of varying salt concentration and the related change in electrolyte conductivity cannot be correctly accounted for.

The nonlinear model of Biesheuvel and Bazant (2010) ${ }^{18}$ captures the dynamics of both the transport on the scale of the whole cell and the charging or discharging of the electric double layer. However, this makes the model rather stiff and difficult to solve especially for large applied voltages. The original approach of ${ }^{18}$ was extended with the modified Donnan model for the description of overlapping electric double layers, ${ }^{19,20}$ other extensions involved accounting for the effect of micropores and tortuosity effects. ${ }^{21,22}$ Recently, Dykstra et al. (2016) included the effect of mesopores into the model. ${ }^{23}$ However, in these models, neither the micropores nor the mesopores are available for transport and these approaches therefore cannot be used to determine and compare the transport limitations.

Therefore, although the models available in the literature together with experimental measurements provide a limited picture about the factors influencing the performance of supercapacitors, different phenomena are described separately or in a simplified manner which lacks the context of the whole system. There is therefore a need for a generalized framework that would allow the different factors that affect the EDLC performance to be compared in a systematic way.

The model presented in this work extends the work of Verbrugge and Liu (2005) with an additional dimension accounting for the trans- 
port in mesopores. This extension enables us to quantitatively compare the transport limitations on the various scales for the first time with a physics-based model. Moreover, the model can simulate the three most commonly used electrochemical characterization techniques (CV, EIS and GC) capturing the specifics of the different modes of operation. Further, various models of the EDL differential capacitance were implemented allowing to account for the potential- or concentrationdependent capacitance, as recently pointed out by various authors. ${ }^{18,24}$

The model was used to quantify the importance of different factors for the performance of EDLCs. The bulk resistance of the electrolyte was identified as the most important factor, while the transport into the particles limited the performance only when the particles were unrealistically large. Furthermore, the specifics related with the use of different techniques to determine the time constant were demonstrated highlighting the necessity to be careful when comparing simulations and measurements of different origin. Finally, a simplified relation allowing for a quick evaluation of the performance of EDLCs is presented and its predictions are compared with the results of the full model. The model is made available for download under a creative commons licence for use as a development tool or to enable the work to be repeated or extended by others.

\section{Mathematical Model}

The model used in this work is based on the porous electrode theory and it builds on previous papers that employed this approach to model the dynamic behavior of supercapacitors. ${ }^{13,14,25}$ In the following section, we first list and discuss the assumptions of the model, then we define the computational domain and derive the model equations based on appropriate balances and finally we introduce different models for the double-layer capacitance.

List of assumptions. - The processes in EDLC take place on wide temporal and spatial scales making it (at least for now) impossible to resolve every detail in one single model. To make the model computationally tractable the following assumptions were adopted:

1. Large cross-section area of electrodes: The dimensions of the EDLC cell are assumed to be much larger in the plane parallel to the electrodes than in the transversal direction (the width and height are larger than the thickness). This assumption is valid for most commercial capacitors ${ }^{26}$ and it allows us to treat the transport in the cell as effectively one-dimensional.

2. Porous-electrode theory: The two phases of a porous medium, i.e., the carbon solid phase and electrolyte liquid phase, are assumed to be perfectly interpenetrating and are volume-averaged in this approach. This assumption is valid as long as the size of pores is significantly smaller than the dimensions of the electrode or separator. A discussion by Verbrugge and Liu (2005) confirmed the validity of this approach for the present system. Furthermore, this assumption enables us to treat the transport into the particles as effectively one-dimensional.

3. Dilute-solution theory: The concentration dependence of the governing parameters except for the ionic conductivity is neglected in this approach. Although this assumption may not be valid for the high concentrations used in this work, this is a commonly adopted simplification because of the increased complexity of concentrated-solution models and due to the fact that a universally accepted model in this field is still missing. ${ }^{24}$

4. Binary, symmetric electrolyte: The electrolyte is assumed to consist of only one pair of ions that carry the same absolute charge and are of the same size. While the first assumption is consistent with electrolytes used in supercapacitors, ${ }^{13}$ the size of both bare and solvated ions usually varies for cations and anions. ${ }^{26,27}$ However, it was shown in a modelling study of Drummond et al. (2015) that the difference in the cell behavior when using symmetric or asymmetric electrolyte is negligibly small. ${ }^{25}$

5. Thin electrical double layer: The thickness of EDL is assumed to be much smaller than the size of pores. This assumption has two main consequences; first, the structure of the EDL does not have

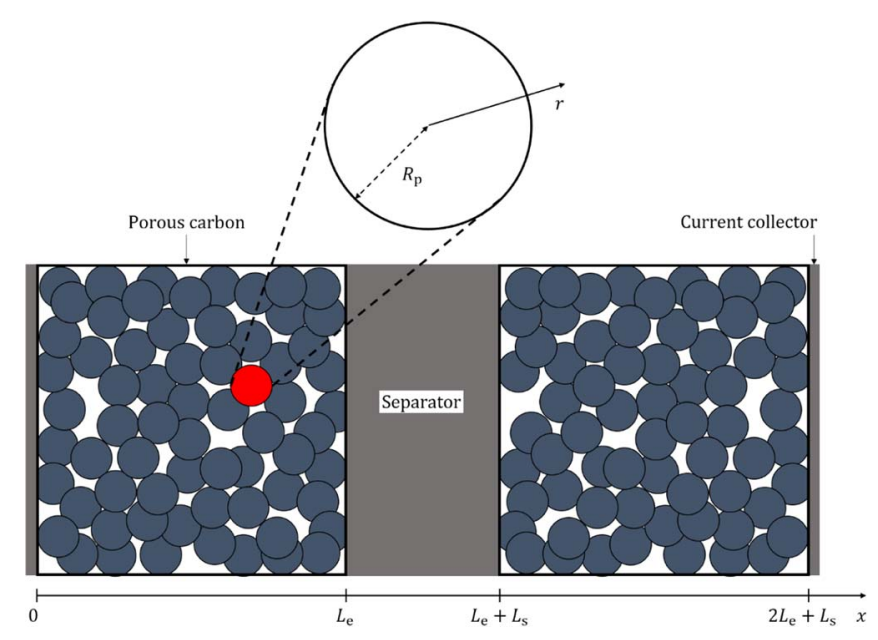

Figure 1. Schematics of the computational domain.

to be resolved in the model and the capacity of the double layer is represented as volume-averaged. The second consequence is that the dynamics of the charging and discharging of the EDL is much faster than other processes such as charge transport on the cell-scale ${ }^{28}$ and it can safely be neglected. This assumption is clearly not valid for the case of micro-pores, where the pore size is comparable to the size of ions. There is, however, no universally accepted theory available for the description of the pore-size dependent capacitance and the dynamics of the desolvation of an ion when entering a micro-pore.

Computational domain.-A typical EDLC consists of two carbon electrodes with a separator in between and two current collectors closing the cell at each side. The electrodes are formed by compressing a carbon particles creating a porous structure. ${ }^{26}$ In this work, the particles were assumed to be uniformly sized spheres with radius $R_{\mathrm{p}}$ as shown in the schematics of the domain in Figure 1.

One-dimensional model.-The one-dimensional (1D) version of the EDLC model was derived by Verbrugge and Liu (2005). In this work, their equations were taken as a starting point and the model is extended with the additional dimension, which corresponds to the transport into the carbon particles. The model resolves both the electrodes and the separator using conservation laws for the charge and mass and employing corresponding constitutional relations for the fluxes.

Under the assumption of a symmetric electrolyte, the balance of charge reduces to the following form ( $\mathrm{se}^{13}$ for derivation):

$$
a C_{\mathrm{D}} \frac{\partial\left(\Phi_{1}-\Phi_{2}\right)}{\partial t}=-\kappa(c) \frac{\partial^{2} \Phi_{2}}{\partial x^{2}},
$$

where $a$ is the specific surface area, $C_{\mathrm{D}}$ is the double layer differential capacitance, $\Phi_{1}$ is the potential in the solid phase, $\Phi_{2}$ is the potential in the electrolyte, $t$ is the time, $\kappa(c)$ is the concentration-dependent ionic conductivity and $x$ is the spatial coordinate (see Figure 1).

The equation for the conservation of the total current flowing through the system can be expressed in various ways, but the formulation of Drummond et al. (2015) is convenient and concise: ${ }^{25}$

$$
0=\sigma \frac{\partial^{2} \Phi_{1}}{\partial x^{2}}+\kappa \frac{\partial^{2} \Phi_{2}}{\partial x^{2}},
$$

where $\sigma$ is the solid-phase conductivity.

Under the assumptions of the bulk electroneutrality, dilute solution theory and symmetric binary electrolyte, the material balance of the electrolyte is given by the following equation:

$$
\epsilon \frac{\partial c}{\partial t}=D \frac{\partial^{2} c}{\partial x^{2}}+\frac{a C_{\mathrm{D}}}{2 F} \frac{\partial\left(\Phi_{1}-\Phi_{2}\right)}{\partial t},
$$

where $\epsilon$ is the electrode porosity, $c$ is the concentration, $D$ is the diffusion coefficient and $F$ is the Faraday constant. 
The separator is not electrically conductive, therefore the descriptions of the charge and mass conservation reduce to the following form:

$$
\begin{gathered}
0=\kappa_{\mathrm{s}}(c) \frac{\partial^{2} \Phi_{2}}{\partial x^{2}}, \\
\epsilon_{\mathrm{s}} \frac{\partial c}{\partial t}=D_{\mathrm{s}} \frac{\partial^{2} c}{\partial x^{2}},
\end{gathered}
$$

where $\kappa_{\mathrm{s}}(c)$ is the concentration-dependent ionic conductivity in the separator, $\epsilon_{\mathrm{s}}$ is the separator porosity and $D_{\mathrm{s}}$ is the diffusion coefficient in the separator.

The transport properties of the porous material for both the charge and mass transport are derived using the effective medium approach. ${ }^{13}$ Therefore, upon defining the free-solution diffusion coefficient $D_{0}$, the effective diffusion coefficient in the electrodes is given by: $D=\frac{D_{0} \epsilon}{\Gamma}$, where $\Gamma$ is the tortuosity of the electrode. The effective diffusion coefficient in the separator is computed as: $D_{\mathrm{s}}=\frac{D_{0} \epsilon_{\mathrm{s}}}{\Gamma_{\mathrm{s}}}$, where $\Gamma_{\mathrm{s}}$ is the tortuosity of the separator. Verbrugge and Liu (2005) showed that the ionic conductivity of uni-univalent electrolyte can be assumed to be a linear function of concentration as follows: ${ }^{13}$

$$
\kappa(c)=\frac{2 F^{2}}{R T} D c,
$$

where $R$ is the universal gas constant and $T$ is the temperature. The ionic conductivity in the separator $\kappa_{\mathrm{s}}(c)$ is obtained from the same equation using $D_{\text {s }}$ instead of $D$.

The boundary conditions effectively summarize the fact that no ions can pass through the current collectors and no electrons can pass through the separator and they are more thoroughly elaborated for the case of the multi-scale model below. Equations 1-5 define a system of partial differential equations with state variables most conveniently defined as: $\Phi_{2}, \Phi_{1}-\Phi_{2}$ and $c$. After a spatial discretization, the system transforms into a set of differential-algebraic equations (DAE) with $\Phi_{2}$ being the algebraic variable. ${ }^{25}$

Multi-scale model.-The 1D model lumps together the properties of the electrode material and electrolyte into a handful of parameters: $C_{\mathrm{D}}, \epsilon, \Gamma, a, \kappa, \sigma$ and $D$, in the framework of the porous electrode theory. However, the activated carbon used as the electrode material contains pores on a broad scale of sizes with very different properties. ${ }^{26}$ To account for this fact, the model is extended with an additional dimension that describes the transport of the charge and mass into and from the carbon particles. In this work, the space between particles is referred to as macro-porous, while the space inside the particles is called meso-porous.

As the electric conductivity of the carbon material is usually much larger than the ionic conductivity of the electrolyte, ${ }^{26}$ it is safe to assume that the solid phase potential $\Phi_{1}$ is constant within the particle. On the other hand, the path for the ions can be more complicated due to a small porosity and large tortuosity of the porous particle. It is therefore assumed that the additional resistance originates from the liquid phase.

The introduction of the additional dimension also extends the number of parameters characterizing the transport properties. The total electrode porosity $\epsilon=\epsilon_{\mathrm{mac}}+\epsilon_{\mathrm{mes}}$ and surface area $a=a_{\mathrm{mac}}+a_{\mathrm{mes}}$ now consist of two contributions of the macro- and meso-pores, respectively. The porosity $\epsilon_{\text {mes }}$ and the specific surface area of mesopores $a_{\text {mes }}$ are related to the total volume of the electrode, i.e., the carbon particles and the space between them. Dividing these quantities by $\left(1-\epsilon_{\mathrm{mac}}\right)$ converts them to the reference frame of the particles only and this is done in the following equations. The differential capacitance $C_{\mathrm{D}}$ is assumed to be the same in both macro- and meso-pores and the tortuosities $\Gamma_{\text {mac }}$ and $\Gamma_{\text {mes }}$ are independent of the total tortuosity $\Gamma$. The effective diffusion coefficients are defined as: $D_{\mathrm{mac}}=\frac{D_{0} \epsilon_{\mathrm{mac}}}{\Gamma_{\mathrm{mac}}}$ and $D_{\text {mes }}=\frac{D_{0} \epsilon_{\text {mes }}}{\Gamma_{\text {mes }}\left(1-\epsilon_{\text {mac }}\right)}$ and ionic conductivities $\kappa_{\text {mac }}$ and $\kappa_{\text {mes }}$ are obtained from Equation 6 in the same way as in the 1D version of the model.

When a particle is sufficiently small, it can be assumed that it is surrounded by an electrolyte with approximately constant potential and concentration. With that assumption, the transport occurs only in the radial direction and the equation for the charge balance in a particle is the following:

$$
\frac{a_{\mathrm{mes}}}{\left(1-\epsilon_{\mathrm{mac}}\right)} C_{\mathrm{D}} \frac{\partial\left(\Phi_{1}-\Phi_{\mathrm{p}}\right)}{\partial t}=-\kappa_{\mathrm{mes}}\left(c_{\mathrm{p}}\right) \frac{1}{r^{2}} \frac{\partial}{\partial r}\left(r^{2} \frac{\partial \Phi_{\mathrm{p}}}{\partial r}\right)
$$

where $\Phi_{\mathrm{p}}$ is the liquid-phase potential in the particle and $r$ is the radial coordinate (see Figure 1). This equation is entirely analagous to Equation 1 with the Laplace operator expressed in spherical coordinates.

The mass balance inside the particle is, again in analogy with the 1D model, described by the following equation:

$\frac{\epsilon_{\mathrm{mes}}}{\left(1-\epsilon_{\mathrm{mac}}\right)} \frac{\partial c_{\mathrm{p}}}{\partial t}=D_{\mathrm{mes}} \frac{1}{r^{2}} \frac{\partial}{\partial r}\left(r^{2} \frac{\partial c_{\mathrm{p}}}{\partial r}\right)+\frac{a_{\mathrm{mes}} C_{\mathrm{D}}}{2 F\left(1-\epsilon_{\mathrm{mac}}\right)} \frac{\partial\left(\Phi_{1}-\Phi_{\mathrm{p}}\right)}{\partial t}$,

where $c_{\mathrm{p}}$ is the salt concentration in the meso-pores.

The charge and mass balances in the macro-porous phase contain an additional term, which corresponds to the flux to or from the particles. This flux is computed from the derivatives at the particle surface and the resulting form of the equations is the following:

$$
a_{\mathrm{mac}} C_{\mathrm{D}} \frac{\partial\left(\Phi_{1}-\Phi_{2}\right)}{\partial t}=-\kappa_{\mathrm{mac}}(c) \frac{\partial^{2} \Phi_{2}}{\partial x^{2}}+\left.a_{\mathrm{mac}} \kappa_{\mathrm{mes}}\left(c_{\mathrm{p}}\right) \frac{\partial \Phi_{\mathrm{p}}(x)}{\partial r}\right|_{r=R_{\mathrm{p}}}
$$

$$
\begin{gathered}
0=\sigma \frac{\partial^{2} \Phi_{1}}{\partial x^{2}}+\kappa_{\mathrm{mac}}(c) \frac{\partial^{2} \Phi_{2}}{\partial x^{2}} \\
\epsilon_{\mathrm{mac}} \frac{\partial c}{\partial t}=D_{\mathrm{mac}} \frac{\partial^{2} c}{\partial x^{2}}+\frac{a_{\mathrm{mac}} C_{\mathrm{D}}}{2 F} \frac{\partial\left(\Phi_{1}-\Phi_{2}\right)}{\partial t}-\left.a_{\mathrm{mac}} D_{\mathrm{mes}} \frac{\partial c_{\mathrm{p}}(x)}{\partial r}\right|_{r=R_{\mathrm{p}}}
\end{gathered}
$$

The separator is assumed to have no capacitance, therefore the potential $\Phi_{\mathrm{p}}$ and concentration $c_{\mathrm{p}}$ in the mesopores are not defined in this domain. The potential $\Phi_{2}$ and concentration $c$ are therefore computed from Equations 4 and 5.

The boundary conditions (BC) reflect the fact that at current collectors $\left(x=0\right.$ and $\left.x=2 L_{\mathrm{e}}+L_{\mathrm{s}}\right)$ all the current is carried by the electrons (potential $\Phi_{1}$ ) as no ions can pass through the collectors. Conversely, only ions are allowed to pass through the separator, therefore the BC express the continuity of fluxes at the boundaries between the electrodes and separator $\left(x=L_{\mathrm{e}}\right.$ and $\left.x=L_{\mathrm{e}}+L_{\mathrm{s}}\right)$. Finally, there is a symmetry BC at the particle center $(r=0)$, while the particle surface $\left(r=R_{\mathrm{p}}\right)$ is surrounded by the bulk electrolyte $\left(\Phi_{2}\right.$ and $\left.c\right)$ at the particular position $x$. In terms of equations, the boundary conditions are defined as follows:

$$
\begin{gathered}
\left.\Phi_{1}\right|_{x=0}=0 \\
\left.\frac{\partial \Phi_{2}}{\partial x}\right|_{x=0}=0 \\
\left.\frac{\partial c}{\partial x}\right|_{x=0}=0 \\
\left.\frac{\partial \Phi_{1}}{\partial x}\right|_{x=L_{\mathrm{e}}}=0 \\
\left.\kappa_{\mathrm{mac}}(c) \frac{\partial \Phi_{2}}{\partial x}\right|_{x=L_{\mathrm{e}}^{-}}=\left.\kappa_{\mathrm{s}}(c) \frac{\partial \Phi_{2}}{\partial x}\right|_{x=L_{\mathrm{e}}^{+}} \\
\left.D_{\mathrm{mac}} \frac{\left.\partial c\right|_{x=L_{\mathrm{e}}^{-}}}{\partial x} D_{\mathrm{s}} \frac{\partial c}{\partial x}\right|_{x=L_{\mathrm{e}}^{+}} \\
\left.\frac{\partial \Phi_{1}}{\partial x}\right|_{x=\left(L_{\mathrm{e}}+L_{\mathrm{s}}\right)}=0
\end{gathered}
$$




$$
\begin{gathered}
\left.\kappa_{\mathrm{s}}(c) \frac{\partial \Phi_{2}}{\partial x}\right|_{x=\left(L_{\mathrm{e}}+L_{\mathrm{s}}\right)^{-}}=\left.\kappa_{\operatorname{mac}}(c) \frac{\partial \Phi_{2}}{\partial x}\right|_{x=\left(L_{\mathrm{e}}+L_{\mathrm{s}}\right)^{+}} \\
\left.D_{\mathrm{s}} \frac{\partial c}{\partial x}\right|_{x=\left(L_{\mathrm{e}}+L_{\mathrm{s}}\right)^{-}}=\left.D_{\mathrm{mac}} \frac{\partial c}{\partial x}\right|_{x=\left(L_{\mathrm{e}}+L_{\mathrm{s}}\right)^{+}} \\
\left.\Phi_{1}\right|_{x=2 L_{\mathrm{e}}+L_{\mathrm{s}}}=U
\end{gathered}
$$$$
\left.\frac{\partial \Phi_{2}}{\partial x}\right|_{x=2 L_{\mathrm{e}}+L_{\mathrm{s}}}=0
$$$$
\left.\frac{\partial c}{\partial x}\right|_{x=2 L_{\mathrm{e}}+L_{\mathrm{s}}}=0
$$$$
\left.\frac{\partial \Phi_{\mathrm{p}}(x)}{\partial r}\right|_{r=0}=0
$$$$
\left.\frac{\partial c_{\mathrm{p}}(x)}{\partial r}\right|_{r=0}=0
$$$$
\left.\Phi_{\mathrm{p}}(x)\right|_{r=R_{\mathrm{p}}}=\left.\Phi_{2}\right|_{x}
$$$$
\left.c_{\mathrm{p}}(x)\right|_{r=R_{\mathrm{p}}}=\left.c\right|_{x}
$$

Models of capacitance.-In the volume-averaged model of a supercapacitor, the EDL is not directly resolved and a model of its capacitance has to be supplied. The capacitance models for interfaces with concentrated electrolytes subjected to a large potential difference were reviewed by Bazant et al. (2009). ${ }^{24}$ In this work, we employed three different models: (i) the Helmholtz model as the simplest approach, which predicts constant capacitance, (ii) the Gouy-ChapmanStern model, which accounts for the dependence of the capacitance on the applied potential difference and (iii) the Bikerman model, which takes into account also the effect of ion crowding and thus reflects both potential and concentration dependence of the capacitance.

The differential capacitance in the Helmholtz model $C_{\mathrm{D}}^{\mathrm{H}}$ is defined by the following relation: ${ }^{26}$

$$
C_{\mathrm{D}}^{\mathrm{H}}=\frac{\epsilon_{\mathrm{r}} \epsilon_{0}}{\lambda_{\mathrm{S}}},
$$

where $\epsilon_{\mathrm{r}}$ is the relative permittivity of the solvent, $\epsilon_{0}$ is the vacuum permittivity and $\lambda_{S}$ is the thickness of the Stern layer, the value of which could be approximated as half of the bare ion diameter.

The potential-dependent differential capacitance in GouyChapman-Stern model $C_{\mathrm{D}}^{\mathrm{GCS}}$ is given as follows: ${ }^{26}$

$$
\frac{1}{C_{\mathrm{D}}^{\mathrm{GCS}}}=\frac{1}{C_{\mathrm{D}}^{\mathrm{H}}}+\frac{1}{C_{\mathrm{D}}^{\mathrm{GC}}},
$$

where the differential capacitance predicted by the Gouy-Chapman model $C_{\mathrm{D}}^{\mathrm{GC}}$ is given by:

$$
C_{\mathrm{D}}^{\mathrm{GC}}=\frac{\epsilon_{\mathrm{r}} \epsilon_{0}}{\lambda_{\mathrm{D}}} \cosh \left(\frac{z e \psi_{\mathrm{D}}}{2 k_{\mathrm{B}} T}\right) .
$$

In this equation, $z$ is the charge number, $e$ is the elementary charge, $k_{\mathrm{B}}$ is the Boltzmann constant, $\psi_{\mathrm{D}}$ is the potential drop across the double layer and $\lambda_{D}$ is the diffuse-layer thickness (Debye length) defined as: ${ }^{24}$

$$
\lambda_{\mathrm{D}}=\sqrt{\frac{\epsilon_{\mathrm{r}} \epsilon_{0} k_{\mathrm{B}} T}{2(z e)^{2} c}} .
$$

For crowded systems, the Bikerman model defines the differential capacitance $C_{\mathrm{D}}^{\mathrm{B}}$ as follows: $:^{24}$

$$
\begin{aligned}
C_{\mathrm{D}}^{\mathrm{B}}= & \frac{\epsilon_{\mathrm{r}} \epsilon_{0}}{\lambda_{\mathrm{D}}} \\
& \times \frac{\sinh \left(\frac{z e\left|\psi_{\mathrm{D}}\right|}{k_{\mathrm{B}} T}\right)}{\left[1+2 \Phi_{\text {bulk }} \sinh ^{2}\left(\frac{z e \psi_{\mathrm{D}}}{k_{\mathrm{B}} T}\right)\right] \sqrt{\frac{2}{\Phi_{\text {bulk }}} \ln \left[1+2 \Phi_{\text {bulk }} \sinh ^{2}\left(\frac{z e \psi_{\mathrm{D}}}{k_{\mathrm{B}} T}\right)\right.}},
\end{aligned}
$$

where $\Phi_{\text {bulk }}=2 a_{\text {sol }}^{3} c N_{\mathrm{A}}$ is the packing parameter and $a_{\text {sol }}$ is the solvated ion diameter. ${ }^{24,28}$

The potential drop $\psi_{\mathrm{D}}$ represents the difference between the potential of the solid phase and the potential in the bulk of the electrolyte. ${ }^{24}$ It is therefore defined as $\psi_{\mathrm{D}}=\Phi_{1}-\Phi_{2}$ for the case of macro-pores and $\psi_{\mathrm{D}}=\Phi_{1}-\Phi_{\mathrm{p}}$ for the case of meso-pores.

Numerical details. - The $x$ and $r$ in the model are coupled only through the fluxes in Equations 9 and 11 and boundary conditions for Equations 7 and 8. Rather than fully two dimensional (2D), the model can be characterized as consisting of two coupled dimensions (1D+1D) on different scales. The set of partial differential equations described by Equations 4, 5 and 7-11 with boundary conditions given by Equations 12-27 was discretized in space using the Finite Volume Method (FVM). This results in a set of differential-algebraic equations with $\Phi_{2}$ being the algebraic variable. The system was numerically integrated in time using the MATLAB solver ode15s and consistent initial conditions were supplied. The spatial discretization step in the $x$-direction was equal to $h=L_{\mathrm{e}} / 30$ and in the $r$-direction the discretization step was equal to $h_{\mathrm{p}}=R_{\mathrm{p}} / 20$. The time-step $\Delta t$ was equal to $0.1 \%$ of the duration of one cycle for the methods introduced in the next section. Simulations with any of the discretization steps reduced to one half lead to only negligible changes in the results and thus the above stated values were considered justified.

\section{Characterization Techniques}

To characterize the performance of supercapacitors, we used three standard electrochemical techniques: the Cyclic Voltammetry (CV), Electrochemical Impedance Spectroscopy (EIS) and Galvanostatic Cycling (GC). In this section, we describe the implementation of these techniques into the model.

As all the methods involve cycling, there is a period at the beginning of each simulation, where the effect of initial conditions is observable. To minimize the effect of initial conditions on the results, the cycling was performed until the change in the value of the cell capacitance (evaluated in each cycle) in two consecutive cycles was smaller than $0.01 \%$ of the maximum (low-frequency) value of the capacitance.

Cyclic voltammetry $(\boldsymbol{C V}) .-\mathrm{CV}$ is a transient technique in which the input voltage $U_{\mathrm{CV}}$ is linearly varied with time creating a cycle over the selected voltage range and the resulting current $I_{\mathrm{CV}}$ is measured. The time-dependent voltage is defined as follows:

$$
U_{\mathrm{CV}}(t)=U_{\max }-v\left|\left(t \bmod 2 t_{0}\right)-t_{0}\right|,
$$

where $\nu$ is the scan rate, $t_{0}=\left(U_{\max }-U_{\min }\right) / \nu$ is the half cycle period and $U_{\max }$ and $U_{\min }$ are the maximum and minimum voltages, respectively. ${ }^{28}$ The charge $Q$ accumulated in the system during one cycle is given by the following expression: ${ }^{28}$

$$
Q=\frac{1}{2} \oint \frac{I_{\mathrm{CV}}}{\nu} \mathrm{d} U_{\mathrm{CV}}
$$

The total capacitance $C_{\mathrm{CV}}$ (in F) can be obtained from: ${ }^{28}$

$$
C_{\mathrm{CV}}=\frac{Q}{\left(U_{\max }-U_{\min }\right)} .
$$


The frequency $f$ of the voltage change in CV simulations is the following:

$$
f=\frac{1}{2 t_{0}}=\frac{v}{2\left(U_{\max }-U_{\min }\right)} .
$$

Electrochemical impedance spectroscopy (EIS).- - In EIS simulations, a sinusoidal input of either the voltage or current with small amplitude is applied to the system. In this work, the input voltage $U_{\text {EIS }}$ was varied according to the following equation: ${ }^{26}$

$$
U_{\text {EIS }}(f)=U_{\text {fix }}+\delta U \exp (j 2 \pi f t),
$$

where $U_{\text {fix }}$ is the fixed voltage and $\delta U$ is the voltage amplitude. By fitting the output current $I_{\text {EIS }}$ by the equation: ${ }^{26}$

$$
I_{\mathrm{EIS}}(f)=I_{\mathrm{fix}}+\delta I \exp [j(2 \pi f t+\Phi)],
$$

the fixed current $I_{\text {fix }}$, current amplitude $\delta I$ and phase angle $\Phi$ can be determined.

Having all these quantities at hand, one can define the complex impedance $Z$ as follows: ${ }^{26}$

$$
Z(f)=\frac{\delta U}{\delta I} \exp (-j \Phi)
$$

The complex capacitance $C_{\mathrm{EIS}}$ of the system is given by:

$$
C_{\mathrm{EIS}}=C^{\mathrm{Re}}-j C^{\mathrm{Im}},
$$

where the real part $C^{\mathrm{Re}}$ of the capacitance is obtained as:

$$
C^{\operatorname{Re}}=\frac{-Z^{\operatorname{Im}}}{2 \pi f|Z|^{2}} .
$$

And the imaginary part $C^{\mathrm{Im}}$ is given by:

$$
C^{\mathrm{Im}}=\frac{Z^{\mathrm{Re}}}{2 \pi f|Z|^{2}},
$$

where $Z^{\mathrm{Re}}$ and $Z^{\mathrm{Im}}$ are the real and imaginary parts of the complex impedance and $|Z|$ is its modulus.

Galvanostatic cycling $(\boldsymbol{G C})$. - GC is a transient technique in which the current is controlled and voltage measured. In our setup, a constant current $I_{\mathrm{GC}}$ was applied to the system starting from the minimum voltage $U_{\min }$ until the maximum voltage $U_{\max }$ was reached. Then the current was reversed, the simulation continued until $U_{\min }$ was reached and then the cycle was repeated. The time $\Delta t_{\mathrm{GC}}$ needed to complete one cycle from $U_{\min }$ to $U_{\max }$ and back was used to compute the capacitance $C_{\mathrm{GC}}$ as follows:

$$
C_{\mathrm{GC}}=\frac{I_{\mathrm{GC}} \Delta t_{\mathrm{GC}}}{2\left(U_{\max }-U_{\min }\right)} .
$$

The frequency of cycling in GC can be defined in two ways. The first involves simply taking the inverse value of $\Delta t_{\mathrm{GC}}$. The disadvantage of this approach is that the frequency cannot be determined a priori and it is the result of the simulation. Moreover, the larger is $\left|I_{\mathrm{GC}}\right|$, the larger is the voltage drop due to ohmic losses upon the reversal of the current, which leads to an increasing overestimation of the frequency for larger currents. For these reasons, we used a corrected frequency $f_{\mathrm{GC}}$ defined as follows:

$$
f_{\mathrm{GC}}=\frac{I_{\mathrm{GC}}}{C_{\max } 2\left(U_{\max }-U_{\min }\right)},
$$

where $C_{\max }$ is the capacitance in the low-frequency limit, which can be a priori computed as:

$$
C_{\max }=\frac{a_{\mathrm{tot}} C_{\mathrm{D}} S L_{\mathrm{e}}}{2} .
$$

To simulate this technique, the boundary condition at the right current collector $\left(x=2 L_{\mathrm{e}}+L_{\mathrm{s}}\right)$ was changed from constant potential (Dirichlet BC) to constant current (Neumann BC) as described in Ref. 13.
Time constant.-The time constant is the characteristic of the dynamic behavior of the supercapacitor. As pointed out by Zhang et al. (2015), several definitions of this quantity exist in the literature. ${ }^{3}$ In this work, the so-called relaxation time constant $\tau_{0}$ was used, which is defined as: ${ }^{3,29}$

$$
\tau_{0}=\frac{1}{f_{0}},
$$

where $f_{0}$ is the frequency at which the capacitance reaches half of its maximum (low-frequency) value. It also corresponds to the maximum of the imaginary part of the capacitance $C^{\mathrm{Im}}$ obtained from the EIS simulations. ${ }^{29}$

\section{Simplified Models}

Although the relatively complex model presented in the previous sections provides a valuable tool for a thorough analysis of the EDLC behavior, the basic response of the system can be captured by a much simpler model. The first simplified model is the RC circuit model, where a resistance and capacitance are connected in series ${ }^{29}$ and the second model introduced by de Levie (1964) is based on the equivalent circuit approach and treats a pore as a network of resistances and capacitances. ${ }^{16}$ These models are used in this work for the comparison with the predictions of the complex model and to establish the limits of the simplified description.

The complex impedance $Z_{\mathrm{RC}}$ of the RC circuit model, i.e., a serial resistance $R$ and capacitance $C_{\max }$ is defined as follows:

$$
Z_{\mathrm{RC}}=R+\frac{1}{j 2 \pi f C_{\max }} .
$$

The real and imaginary capacitance are then extracted entirely analogically to the procedure described for the Electrochemical Impedance Spectroscopy.

The de Levie model is used to compute the complex impedance $Z_{\mathrm{p}}$ of the porous electrode as follows: ${ }^{16}$

$$
Z_{\mathrm{P}}=R_{\mathrm{I}} \frac{\operatorname{coth} \sqrt{j 2 \pi f R_{\mathrm{I}} C_{\mathrm{max}}}}{\sqrt{j 2 \pi f R_{\mathrm{I}} C_{\max }}},
$$

where $R_{\mathrm{I}}$ is the resistance of the pores. The total impedance $Z_{\mathrm{EC}}$ of the cell described by this equivalent circuit model is given by the following equation:

$$
Z_{\mathrm{EC}}=Z_{\mathrm{P}}+R_{\mathrm{s}},
$$

where $R_{\mathrm{S}}$ is the resistance of the separator. In these models, the capacitance $C_{\max }$ is computed as the low-frequency limiting (maximum) capacitance, see Equation 45 . The resistance of the pores $R_{\mathrm{I}}$ is the combined resistance of the solid phase and electrolyte in the electrodes. For this case, it was demonstrated by various authors ${ }^{8,10,15}$ that the overall conductivity $\tilde{\kappa}$ of the porous electrode can be expressed as follows:

$$
\tilde{\kappa}=\frac{2 \kappa \sigma}{\kappa+\sigma} .
$$

The resistance $R_{\mathrm{I}}$ is then defined as $:^{8}$

$$
R_{\mathrm{I}}=\frac{2 L_{\mathrm{e}}}{\tilde{\kappa} S} .
$$

Analogically, the resistance of the separator is given by:

$$
R_{\mathrm{s}}=\frac{L_{\mathrm{s}}}{\kappa_{\mathrm{s}} S} .
$$

And the total resistance is $R=R_{\mathrm{I}}+R_{\mathrm{S}}$.

Generalized relation.-Let us now develop an alternative approach for the determination of the frequency-dependent capacitance in the RC circuit. When a voltage step $\Delta U$ is applied to this system, which was initially at zero volts, the actual voltage of the resistor and 

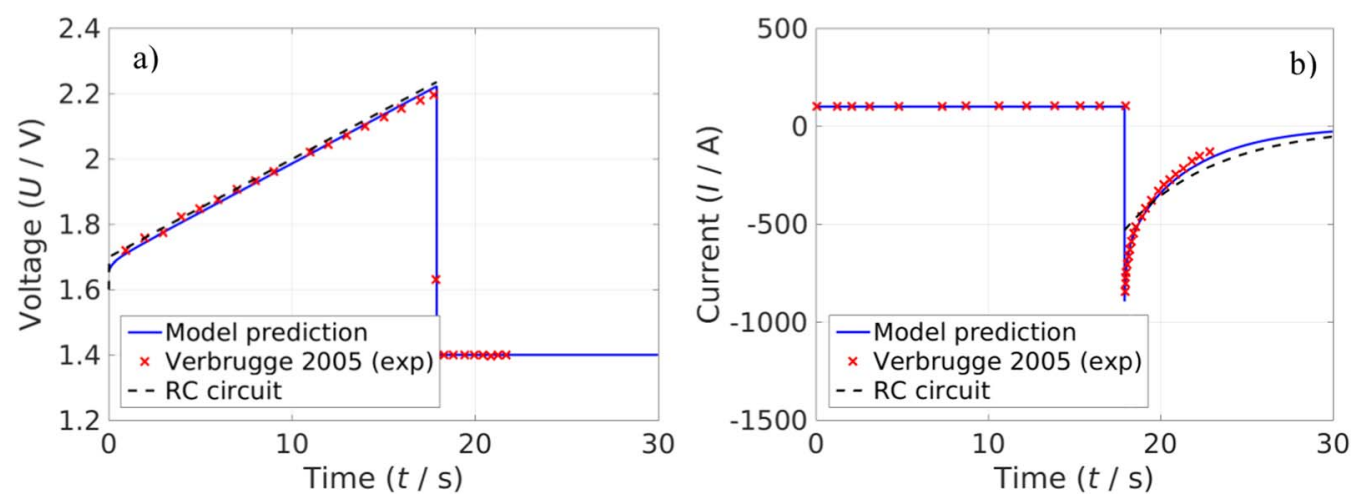

Figure 2. a) Voltage and b) current as a function of time. The model prediction is compared with experimental data of Verbrugge (2005) and the prediction of the RC circuit model. The specific surface area was $a=4.5 \cdot 10^{7} \mathrm{~m}^{2} / \mathrm{m}^{3}$.

the capacitor changes over time. The time-development of the voltage of the capacitor $U_{\mathrm{C}}$ is described by the following function:

$$
U_{\mathrm{C}}=\Delta U\left[1-\exp \left(-\frac{t}{R C_{\max }}\right)\right] .
$$

If we multiply the whole equation by $C_{\max }$ and divide by $\Delta U$, we obtain on the left-hand side the charge of the capacitor $Q_{\mathrm{C}}=C_{\max } U_{\mathrm{C}}$ divided by the total voltage diference. By replacing $t$ with the characteristic time $\tau$ of the mode at which the system should operate (e.g., for EIS the characteristic time would be $\tau=1 / 2 \pi f$ ), the quantity $Q_{\mathrm{C}} / \Delta U$ on the left-hand side in fact becomes the accesible (or frequency-dependent) capacitance $C(f)$ for the process:

$$
C(f)=\mathrm{C}_{\max }\left[1-\exp \left(-\frac{\tau}{R C_{\max }}\right)\right] .
$$

Furthermore, the time constant $\tau_{0}=1 / f_{0}$, which corresponds to the frequency $f_{0}$ at which the accessible capacitance $C(f)$ is equal to $\mathrm{C}_{\max } / 2$ can be derived as:

$$
\tau_{0}=2 \pi \ln (2) R \mathrm{C}_{\max } .
$$

And by plugging in the expressions for the resistance and capacitance, the final equation is obtained:

$$
\tau_{0}=\pi \ln (2) a C_{\mathrm{D}} L_{\mathrm{e}}\left(\frac{2 L_{\mathrm{e}}}{\tilde{\mathrm{\kappa}}}+\frac{L_{\mathrm{s}}}{\kappa_{\mathrm{s}}}\right),
$$

which is a direct function of the construction and physical parameters of the cell.

\section{Results and Discussion}

Constant current charge - constant voltage discharge (CCC$\boldsymbol{C V D}$ ).-Before proceeding to the performance evaluation of supercapacitors, let us compare the predictions of our model to the experimental data of Verbrugge and Liu (2005). ${ }^{13}$ In these experiments, the constant current $(I=100 \mathrm{~A}$ ) charging was followed by the discharge at constant voltage $(U=1.4 \mathrm{~V})$. The comparison is shown in Figure 2 and these results were obtained with the $1 \mathrm{D}$ version of the model in order to obtain a setup comparable to the original paper. The good agreement between the model prediction and the experimental data is comparable to different modelling studies that used the same experimental data for validation. ${ }^{13,14,25}$

It is worth noting that the simple RC circuit model reproduces the charge-discharge profile only relatively well. While the constant current charge is captured very well, the discharging dynamics under constant voltage is reproduced poorly. This is due to the fact that the $\mathrm{RC}$ circuit model does not take into account the spatial distribution of potential in the cell. In reality and in the complex model, after the abrupt change in the voltage the potential difference $\Phi_{1}-\Phi_{2}$ initially quickly changes only in the regions close to the current collectors and the separator (see Figure 3). This leads to the fact that the cell initially appears to have lower resistance and capacitance, hence the initial discharge current is larger and decays more quickly.

The spatial distributions for the CCC-CVD simulation are shown in Figure 3. During the constant current charging, both the concentration and potential are changing steadily after an initial transient period. This period is caused by the electrolyte resistance. On the other hand, the spatial distributions for constant voltage discharge show more complicated behavior. There is an abrupt change in the voltage, which is reflected by the sharp decrease in the separator region (between 50 and 75 micrometers). The fact that the cell starts suddenly discharging leads to a quick change in the concentration, which however takes place only in the electrode and it takes some time for the concentration in the separator to adapt to this trend.

Methods for evaluation of performance.-The performance of an EDLC can be assessed by a variety of methods, each of which has its specific advantages and disadvantages. For the sake of simplicity, the results in this section were once again obtained with the $1 \mathrm{D}$ version of the model. The effect of mesopores is addressed later in a dedicated section.

The performance of an EDLC is a function of the time-scale at which the device operates. This is demonstrated in Figure 4, where the cyclic voltammograms for different values of scan rate are shown. The shape of the curve changes from an almost rectangular at a slow scan rate, which is typical for a capacitor, to almost linear for a fast scan rate, which is characteristic for a resistor.

A similar change occurs in the plot showing the results of EIS simulations that is shown in Figure 5. Please note that compared to $\mathrm{CV}$ simulations, there is much narrower potential window and also the curves are smoother due to the different nature of the excitation signal.

In Galvanostatic Cycling, the voltage response of the system changes with increasing current as can be seen from Figure 6. The higher is $I$, the larger is the step change in voltage after current reversal. Eventually, for large currents this step change that can be ascribed to the ohmic drop in the system covers the whole potential window leading to the capacitance equal to zero.

It is clear that the performance of the EDLC is a strong function of the operating parameters of the characterization methods. This is expressed in Figure 7 in the plots relating the capacitance to frequence and current. It is worth noting that the capacitance predicted by EIS decays at larger frequencies than in CV simulations in Figure 7a. This effect can be ascribed to the much narrower voltage window and thus smaller concentration changes in EIS and also to its smoother excitation signal.

Effect of electrode thickness.-The thickness of the electrode $L_{\mathrm{e}}$ is one of the basic construction parameters of the EDLC cell. It has a twofold effect on the cell behavior; first, by increasing the thickness, the ionic resistance of the electrode increases and second, 

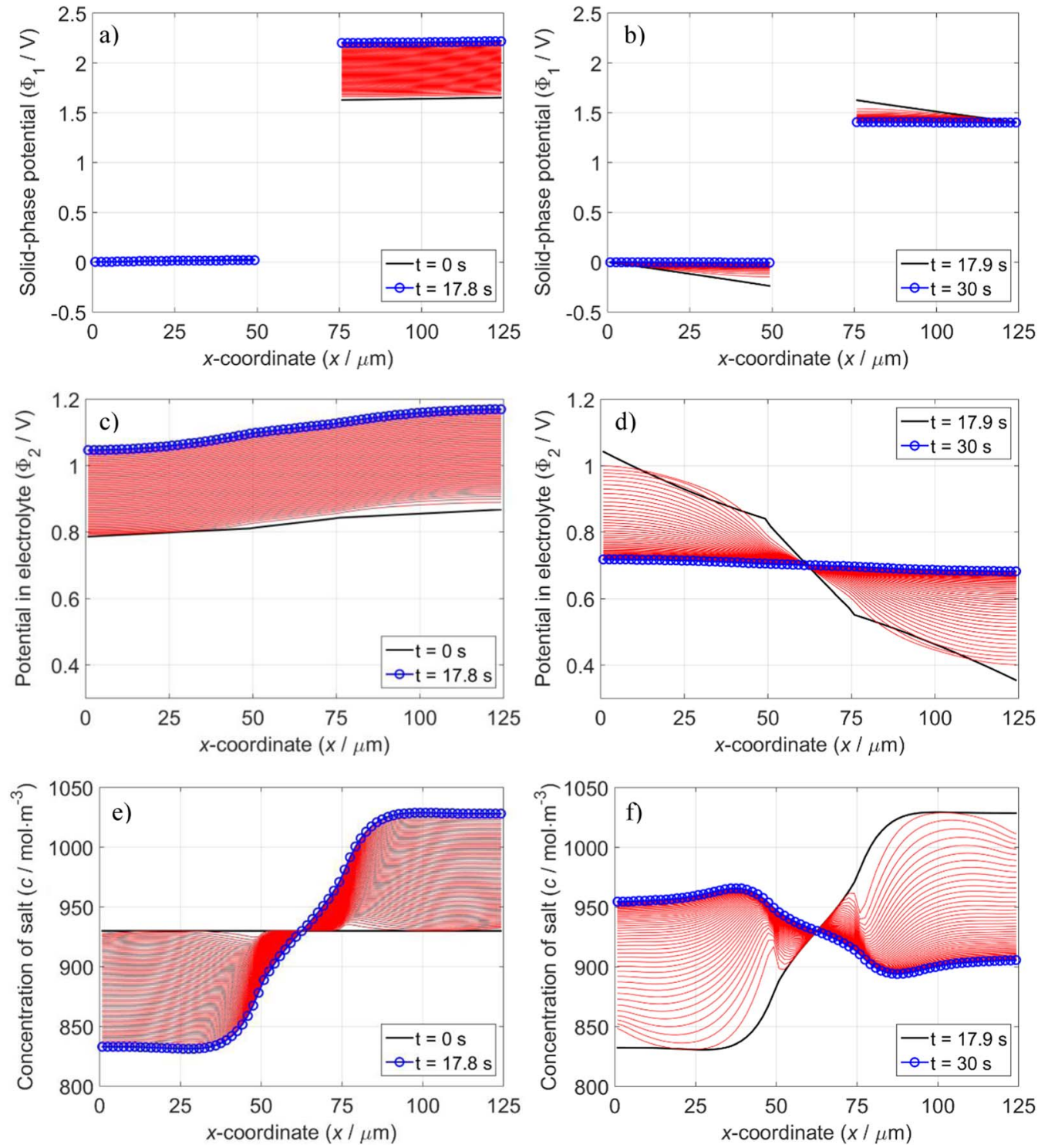

Figure 3. Spatial distribution of (a,b) solid phase potential, $(\mathrm{c}, \mathrm{d})$ potential in electrolyte and $(\mathrm{e}, \mathrm{f})$ salt concentration during constant current charging (a,c,e) and constant voltage discharging $(\mathrm{b}, \mathrm{d}, \mathrm{f})$.
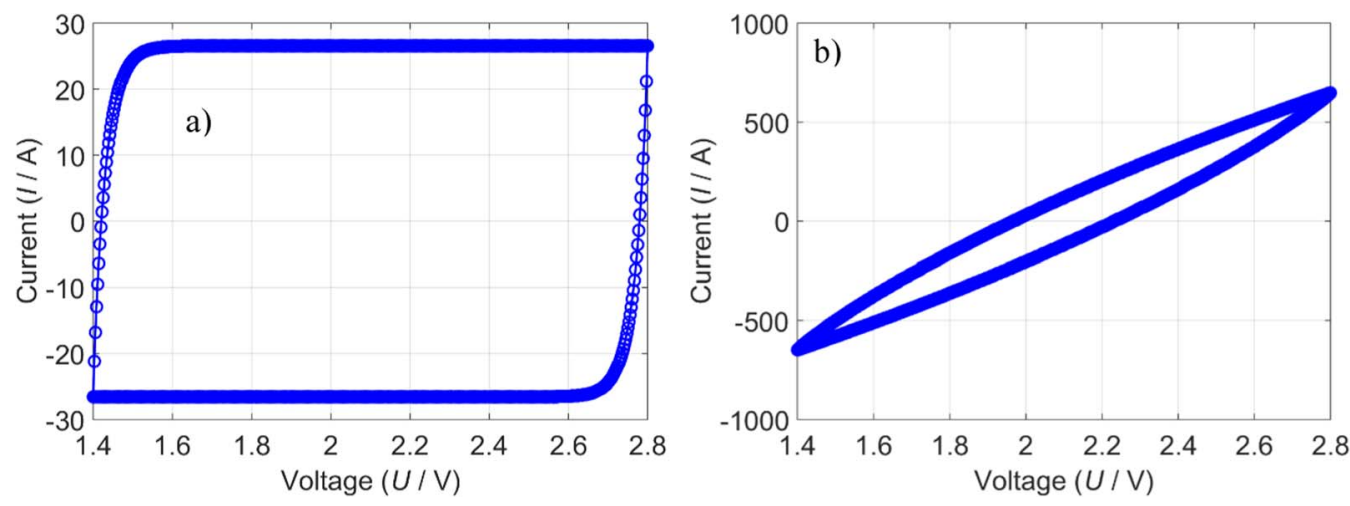

Figure 4. Cyclic voltammetry profiles for scan rates a) $v=0.01 \mathrm{~V} \cdot \mathrm{s}^{-1}$ and b) $v=1 \mathrm{~V} \cdot \mathrm{s}^{-1}$. 

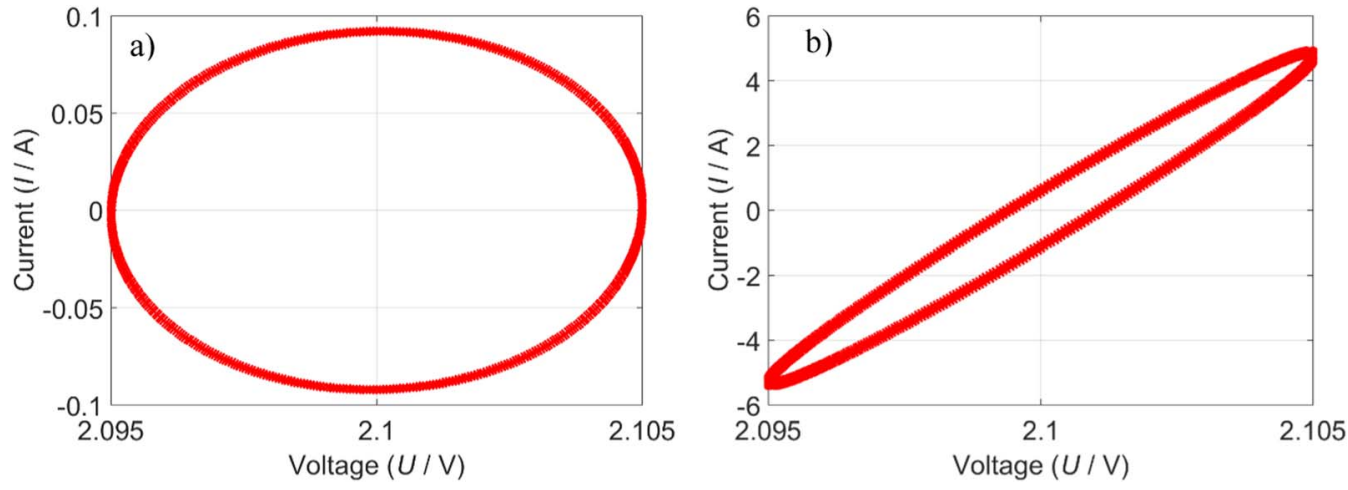

Figure 5. Electrochemical impedance spectroscopy profiles for frequencies a) $f=0.001 s^{-1}$ and b) $f=1 s^{-1}$.
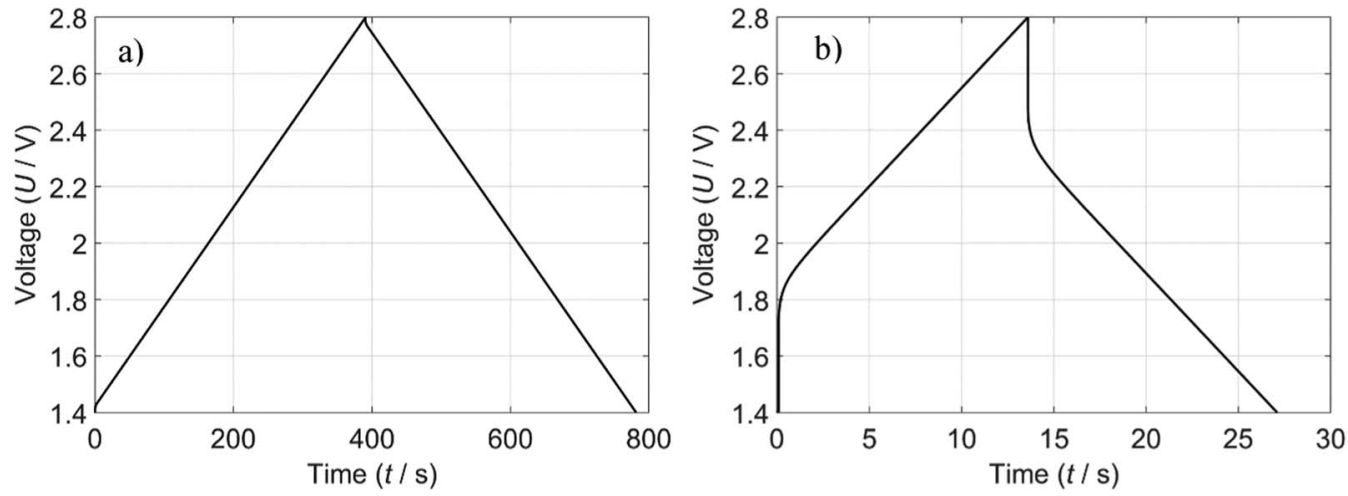

Figure 6. Galvanostatic cycling profiles for currents a) $I=10 \mathrm{~A}$ and b) $I=200 \mathrm{~A}$.
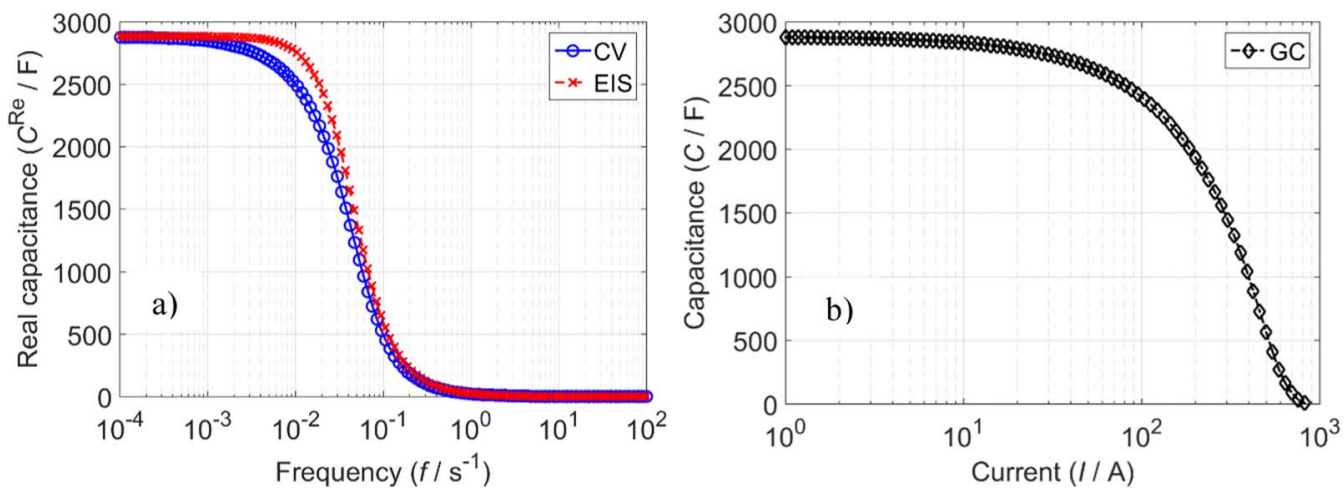

Figure 7. a) Capacitance as a function of frequency obtained from Cyclic Voltammetry and Electrochemical Impedance Spectroscopy simulations. b) Capacitance as a function of current from Galvanostatic Cycling simulations.
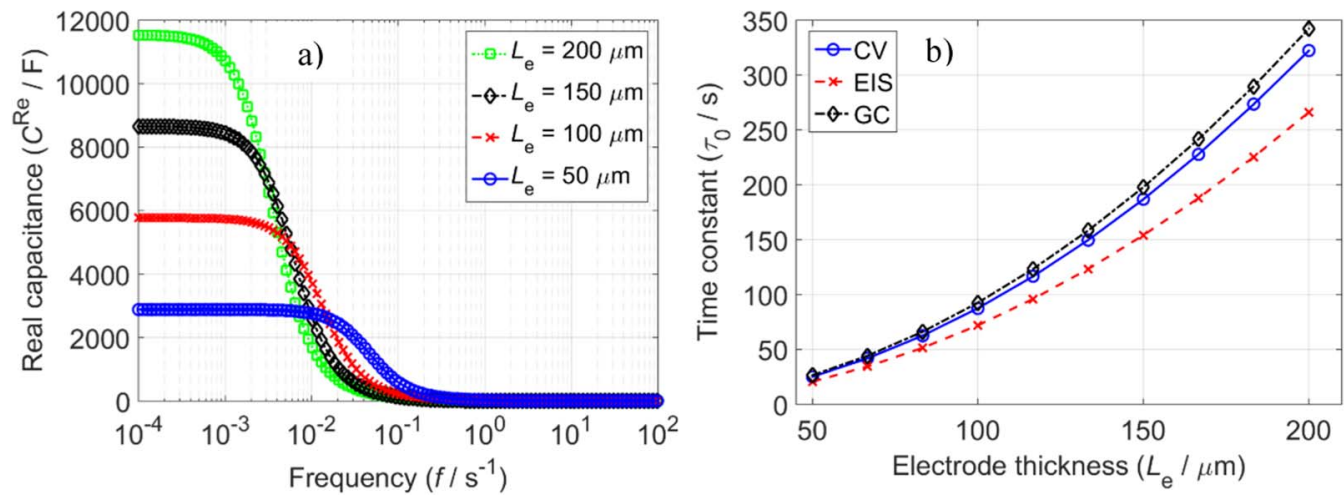

Figure 8. (a) The real part of the complex capacitance as a function of frequency for different values of electrode thickness $L_{e}$. (b) The time constant as a function electrode thickness as predicted by different methods: Cyclic Voltammetry (CV), Electrochemical Impedance Spectroscopy (EIS) and Galvanostatic Cycling (GC). 

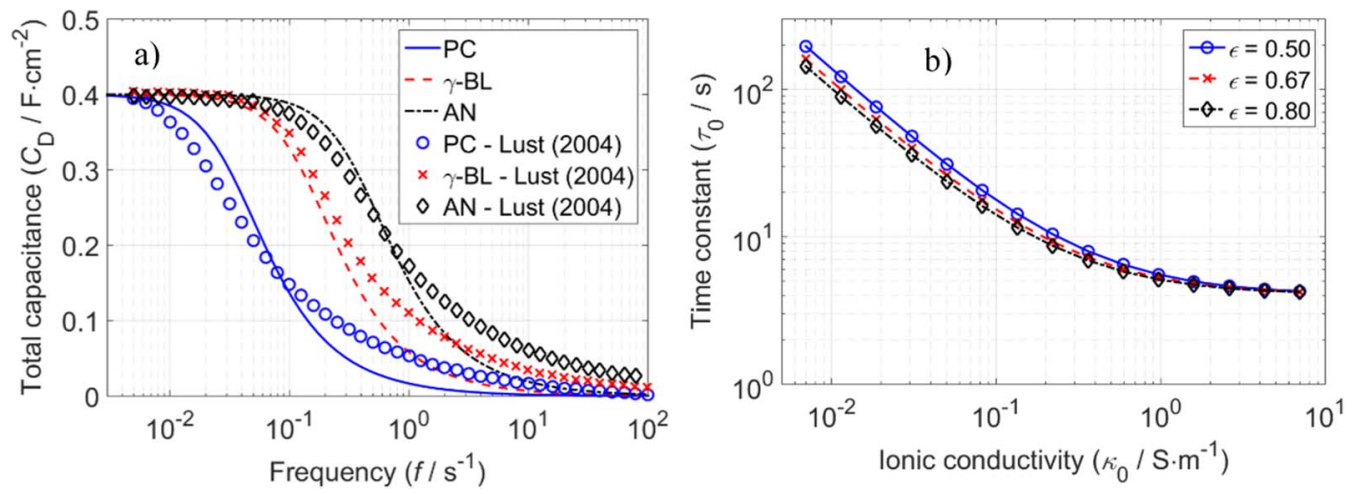

Figure 9. (a) Total capacitance as a function of frequency for different solvents: acetonitrile (AN), $\gamma$-butyrolactone ( $\gamma$-BL) and propylene carbonate (PC). The experimental data represented by symbols were taken from Lust (2004). ${ }^{8}$ The parameters of the simulations were the following: $L_{e}=100 \mu \mathrm{m}, a=8 \cdot 10^{7} \mathrm{~m}^{2} / \mathrm{m}^{3}$, $S=6.45 \cdot 10^{-3} m^{2}, \kappa_{P C}=0.057 \mathrm{~S} \cdot \mathrm{m}^{-1}, \kappa_{\gamma-B L}=0.29 \mathrm{~S} \cdot \mathrm{m}^{-1}, \kappa_{A N}=0.64 \mathrm{~S} \cdot \mathrm{m}^{-1}, \sigma_{P C}=0.23 \mathrm{~S} \cdot \mathrm{m}^{-1}, \sigma_{\gamma-B L}=0.58 \mathrm{~S} \cdot \mathrm{m}^{-1}, \sigma_{A N}=2.7 \mathrm{~S} \cdot \mathrm{m}^{-1}$ and $\Gamma=1.15$. (b) Time constant as a function of solvent conductivity (free solution) for base case parameters and for different values of porosity $\epsilon$.

as the volume of the electrode is increased, the capacitance grows proportionally. Both these effects can be seen in the EIS simulation results for different values of $L_{\mathrm{e}}$ in Figure 8a. As a consequence, the time constant $\tau_{0}$ increases with the square of $L_{\mathrm{e}}$ and as shown in Figure $8 \mathrm{~b}$ this scaling is predicted similarly by different methods. Unlike the scaling, the value of time constant for a given electrode thickness varies significantly with the used method and the difference is as large as $26 \%$ between Electrochemical Impedance Spectroscopy and Galvanostatic Cycling.

These discrepancies can be ascribed to the rather different operation conditions of the methods. In EIS the voltage window is very narrow and changes in the input signal are small leading to small concentration changes and the smallest predicted time constant. On the other hand $\mathrm{CV}$ and $\mathrm{GC}$ as dynamic methods cover much larger voltage ranges leading to larger changes in concentration and thus conductivity and this results in the larger values of $\tau_{0}$ compared to EIS. Moreover, in GC, the current direction is switched abruptly from positive to negative and vice versa, which leads to larger losses in the system and even further increase of the time constant when compared to $\mathrm{CV}$.

Effect of electrolyte conductivity.-The conductivity of the electrolyte $\kappa$ is one of the determining factors for the ionic resistance of the cell. The choice of solvent has a large effect on $\kappa$ due to the solvent permittivity and transport properties. EIS simulations for three different solvents were performed and the results are shown in
Figure 9a, where they are compared to the experimental data of Lust et al. (2004). ${ }^{8}$ The parameters of the model were chosen to resemble the conditions of the experiments and only those that are different from the base case are listed in the figure caption. It can be seen that for larger conductivity the curves shift to higher values of frequency and there is a good agreement between the model predictions and experimental results.

To further generalize this important result, the dependence of the time constant on the ionic conductivity is depicted in Figure 9b. For low values of $\kappa_{0}$, it results in a straight decreasing line in the double logarithmic plot and because the slope of the line is equal to -1, there is clearly an inverse proportionality between $\tau$ and $\kappa_{0}$. The effect of porosity in the same figure is not negligible, but relatively small compared to the effect of conductivity. For larger values of the ionic conductivity, all the curves reach a plateau and do not decrease further. This is due to the fact that the solid-phase conductivity $\sigma$ is relatively small and the resistance of the solid phase becomes determining for the cell when $\kappa_{0}$ is large.

Effect of separator properties. - The separator acts as a resistance for the ionic current in the cell preventing the electrodes from contacting while allowing the ions to pass through. As such, it is desirable that the ionic resistance of the separator is the smallest possible. The results of EIS simulations for different values of separator thickness are shown in Figure 10a and the predictions are compared with the experimental results of Tõnurist et al. (2005). ${ }^{10}$ The parameters of the
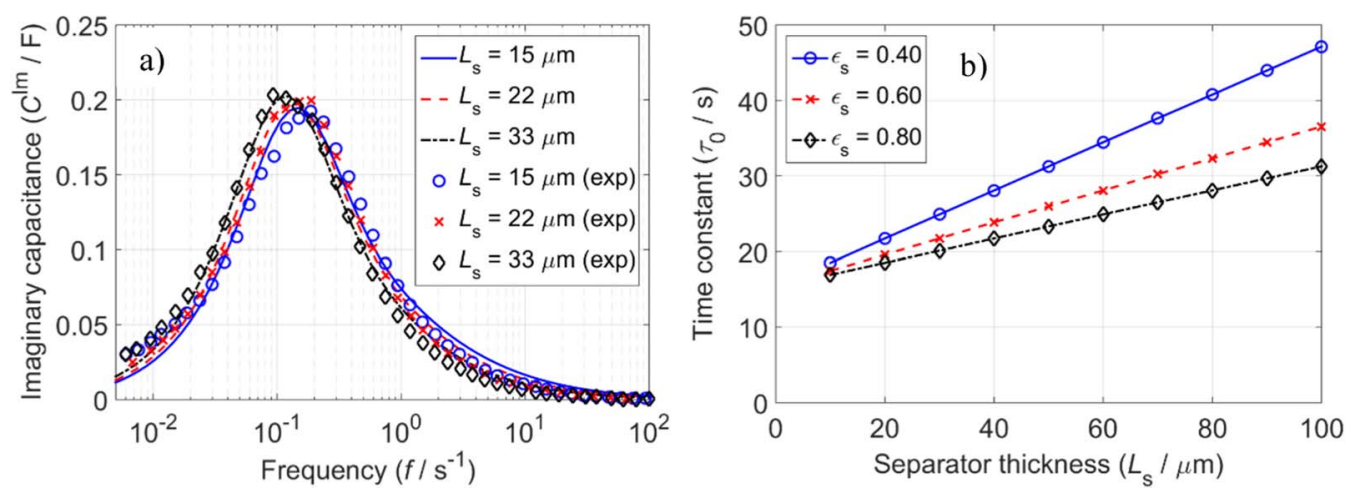

Figure 10. (a) Imaginary part of the complex capacitance as a function of frequency for different values of the separator thickness. The experimental data represented by symbols were taken from Tõnurist (2009). ${ }^{10}$ The parameters of the simulations were the following: $L_{e}=120 \mu \mathrm{m}, a C_{D}=72.4 \cdot 10^{7} \mathrm{~F} / \mathrm{m}^{3}$, $S=0.2 \cdot 10^{-3} \mathrm{~m}^{2}, \kappa_{A N}=1.04 \mathrm{~S} \cdot \mathrm{m}^{-1}, c_{0}=1000 \mathrm{~mol} \cdot \mathrm{m}^{-3}, \Gamma=\Gamma_{s}=1.29$ and $\epsilon_{s}=0.42$. (b) Time constant as a function of separator thickness for base case parameters and for different values of separator porosity $\epsilon_{s}$. 

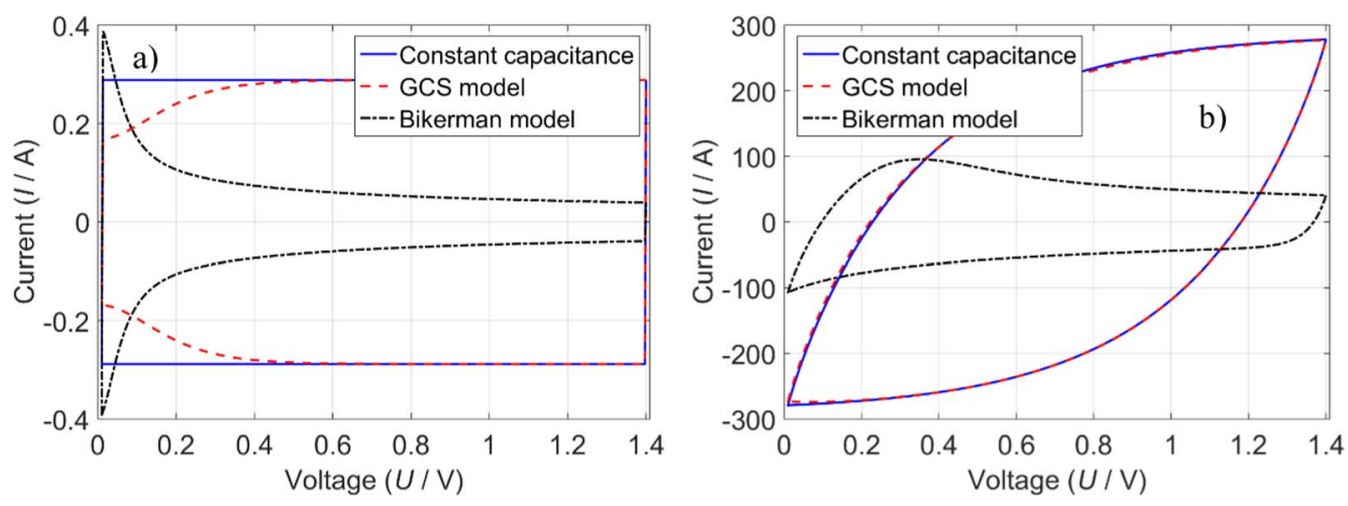

Figure 11. Current as a function of voltage from Cyclic Voltammetry simulations for different EDL models. The scan rates were: a) $v=1 \cdot 10^{-4} V \cdot s^{-1}$ and b) $v=0.1 \mathrm{~V} \cdot \mathrm{s}^{-1}$.

simulations are given in the caption and they were chosen to resemble the experimental conditions as closely as possible. The only adjusted parameter was the tortuosity which was assumed the same in electrode and in separator and was equal to $\Gamma=\Gamma_{\mathrm{s}}=1.29$.

The results are further generalized in Figure $10 \mathrm{~b}$ where the dependence of the time constant on the separator thickness for different values of separator porosity are plotted. It can be seen that the dependence results in straight lines, which corresponds with the fact that the separator acts as an additional ionic resistance. As expected, the increasing separator porosity reduces the time constant by lowering the separator resistance.

Models of electric double layer.-Three different models of the EDL capacitance were used to demonstrate different effects: the Helmholtz model (constant capacitance), Gouy-Chapman-Stern model (potential-dependent) and Bikerman model (ion crowding). The results of $\mathrm{CV}$ simulations with different EDL models are shown in Figure 11 for two different values of scan rate and voltage window between 0 and 1.4 volts. In this voltage range and for low scan rate (Figure 11a), there are clear differences between the predictions of the different models, namely, the increase of capacitance between 0 and 0.4 volts predicted by the GCS model and the gradual decrease of capacitance with increasing voltage predicted by the Bikerman model. With increasing scan rate (see Figure 11b) these differences become blurred by the transport limitation effects discussed in previous sections.

This effect is reflected in the dependence of capacitance on scan rate for different EDL models presented in Figure 12. It shows that

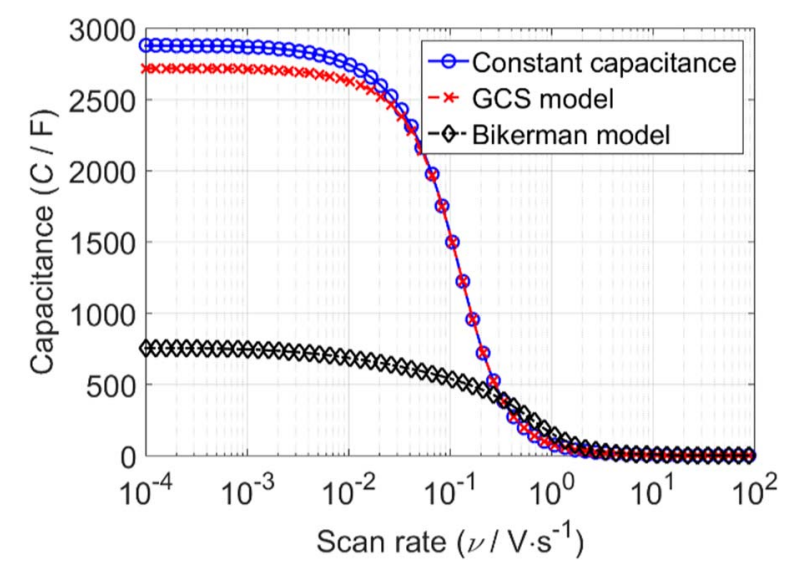

Figure 12. Capacitance as a function of scan rate for different models of EDL capacitance. while the low-frequency capacitance is predicted differently by each of the models, the high-frequency decreace of capacitance remains almost unchanged.

Although it is clear that different physical phenomena represented by the three different EDL capacitance models affect the supercapacitor behavior in the region around zero volts, commonly used devices rarely operate under these conditions. However, in the voltage window between 1.4 and 2.8 volts, which is typical for the operation of supercapacitors and was also used in most of this work, all the EDL capacitance models provide constant (or nearly constant for the Bikerman model) value of capacitance (also apparent in Figure 11a). While the Helmholtz and GCS models agree in the value of capacitance for high voltages, the Bkerman model predicts much smaller values. This is probably caused by the crowding effect, which is very strong for the concentrated salt considered in this work. Nevertheless, the actual value of the EDL capacitance is usually a fitting parameter for models on the device-scale. Therefore, the most important conclusion of this analysis is that even when taking into account various phenomena affecting the capacitance of a planar EDL, there is only a very small difference between the predictions of the commonly used EDL models in the voltage range typically used in commercial devices. The constant capacitance was assumed for all studies in this work except for this section. Although the use of the constant capacitance model is well justified for our case of high salt concentration (i.e., thin EDL), different approaches are needed when the diffuse part of the EDL is important. Particularly, the difference between ion adsorption and salt adsorption becomes more pronounced and it needs to be taken into account. A framework for the modelling of this phenomenon was introduced by Biesheuvel and Bazant (2010). ${ }^{18}$

Experimentally, the capacitance is observed to be dependent on the cell potential even in the aforementioned typical voltage range. ${ }^{1,30}$ However, this effect is highly dependent on the specific system under consideration and clearly it cannot be easily described using the commonly used EDL models. More elaborate approaches such as the molecular dynamics are necessary to explain these phenomena.

Effect of mesopores. - The multi-scale model allows us to treat separately the transport of ions between the carbon particles, i.e., in macro-pores and inside of these particles, i.e., in meso-pores. With this approach, it is possible to identify the limiting factors for given parameters. In Figure 13, the time constant obtained from EIS simulations is plotted as a function of the particle radius for different ratios of macro- to meso-porosity, while the total porosity was the same for all the simulations. Clearly, there is a certain threshold value of $R_{\mathrm{p}}$ below which the limitation of the transport inside the particles is negligible. This threshold value is smaller the smaller is the meso-porosity due to the fact that $\epsilon_{\text {mes }}$ effectively reduces the diffusion coefficient and conductivity inside the particles. Similarly, the value of $\tau_{0}$ at the plateau for small $R_{\mathrm{p}}$ is reduced for larger $\epsilon_{\mathrm{mac}}$, which is entirely the same 


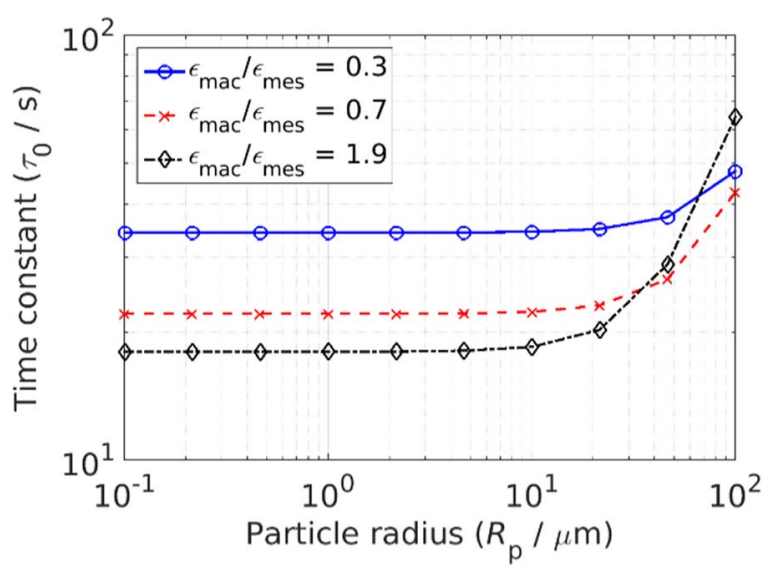

Figure 13. Time constant as a function of the carbon particle radius for different ratios of the macropore porostiy $\epsilon_{\text {mac }}$ and porosity of the particles $\epsilon_{\text {mes }}$.

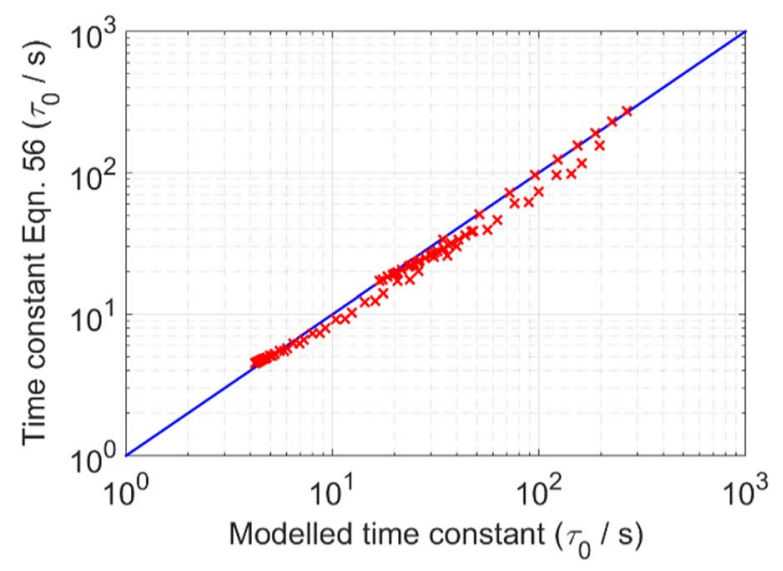

Figure 15. Comparison of the time constant predicted by Equation 56 with the time constant obtained by the complex model using EIS from the parametric studies of $L_{e}, \kappa_{0}, L_{s}, \epsilon$ and $\epsilon_{s}$.

behavior, although they carry the majority of the surface area and thus capacity of the cell.

Comparison of models.-Predictions of different models are compared in Figure 14 in the Nyquist plot of capacitance and in the plot of frequency-dependent real capacitance. The RC circuit model as the most idealized provides a symmetric plot in the $C^{\mathrm{Im}}-C^{\mathrm{Re}}$ plot, while the model used in this work and the de Levie model predict curves that are deformed. This is due to the fact that they both take into account the spatial distribution of potential in the electrodes and separator. On the other hand the de Levie model neglects the concentration changes and the resistance in the solid phase. From the results in Figure 14, the RC circuit model and the de Levie model can be viewed as limiting cases, while the prediction of the more complex model lies in between. On the other hand, the prediction of Equation 54, which was derived from the step-change of voltage in the $\mathrm{RC}$ circuit, agrees with the results of the complex model remarkably well.

The accuracy of the value of $\tau_{0}$ predicted by Equation 56 is assessed in Figure 15 by the comparison to the predictions of the complex model for the parametric studies of $L_{\mathrm{e}}, \kappa_{0}, L_{\mathrm{s}}, \epsilon$ and $\epsilon_{\mathrm{s}}$. A straight line in this plot relating the two predictions corresponds to a perfect agreement. Although part of the points lie well below the straight line, it is possible to say that the prediction of Equation 56 agrees well with the results of the complex model in terms of both the scaling and the absolute values. Given the relative simplicity of Equation 56, it
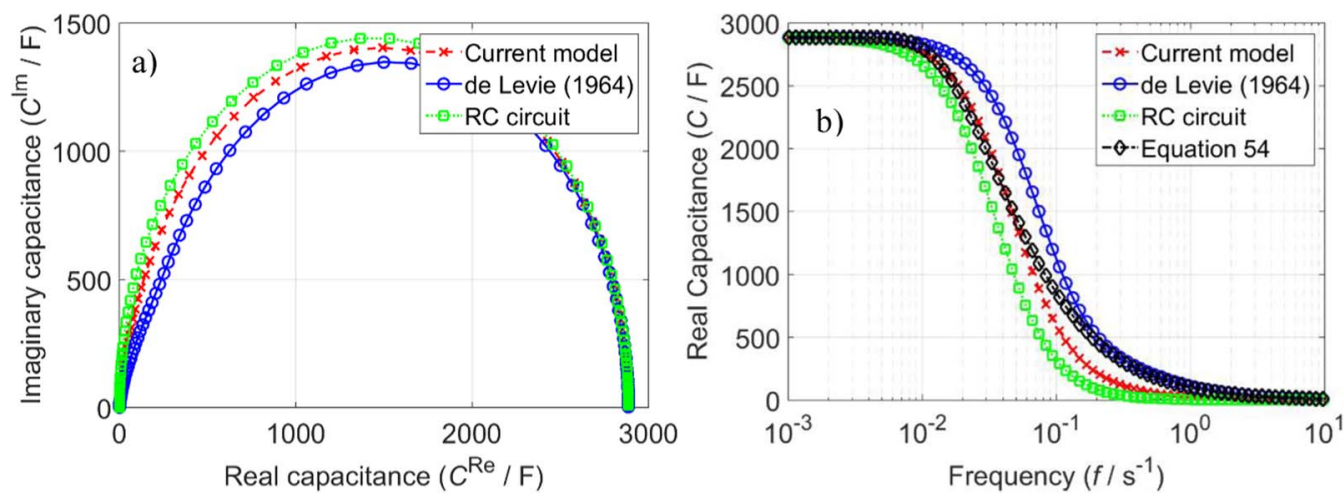

Figure 14. (a) Nyquist plot of complex capacitance and (b) the real capacitance as a function of frequency as predicted by different models. 
provides a good tool for the quick evaluation of the time constant for supercapacitors.

\section{Conclusions}

A dynamic multi-scale model of an Electric Double Layer Capacitor (EDLC) was developed and used for the evaluation of the EDLC performance. The performance was described by the time constant $\tau_{0}$, which characterizes the decrease of the capacitance at high frequencies. In an agreement with experimental studies, the time constant was shown to increase with increasing thickness of the electrodes and separator leading to a poorer performance. On the other hand, $\tau_{0}$ decreased with increasing ionic conductivity of the electrolyte, but only until the point, where the resistance of the solid phase (i.e., the carbon electrode) became important. The assumption of capacitance independent of voltage was shown to be well justified unless the cell was close to its total discharge, at least as predicted by standard double-layer capacitance models. The resistance of the meso-pores inside the car- bon particles was demonstrated to be negligible for the typical sizes of particles around one micrometer. On the other hand, when the voltage window was large, salt depletion was observed in the meso-pores leading to poorer performance. To the best of our knowledge, such findings obtained with a physics-based model are presented for the first time. Finally, a generalized equation was derived that is able to reproduce the results and trends predicted by the complex model with reasonable accuracy. As such, the models presented in this work can serve both for a quick evaluation of the performance of new devices and for thorough inspection of more complex phenomena taking place in supercapacitors.

\section{Acknowledgment}

This work was financially supported by a specific university research grant (CZ MSMT No 20/2016). Wasim Sarwar is gratefully acknowledged for very helpful discussions.

\section{List of Symbols and Parameter Values}

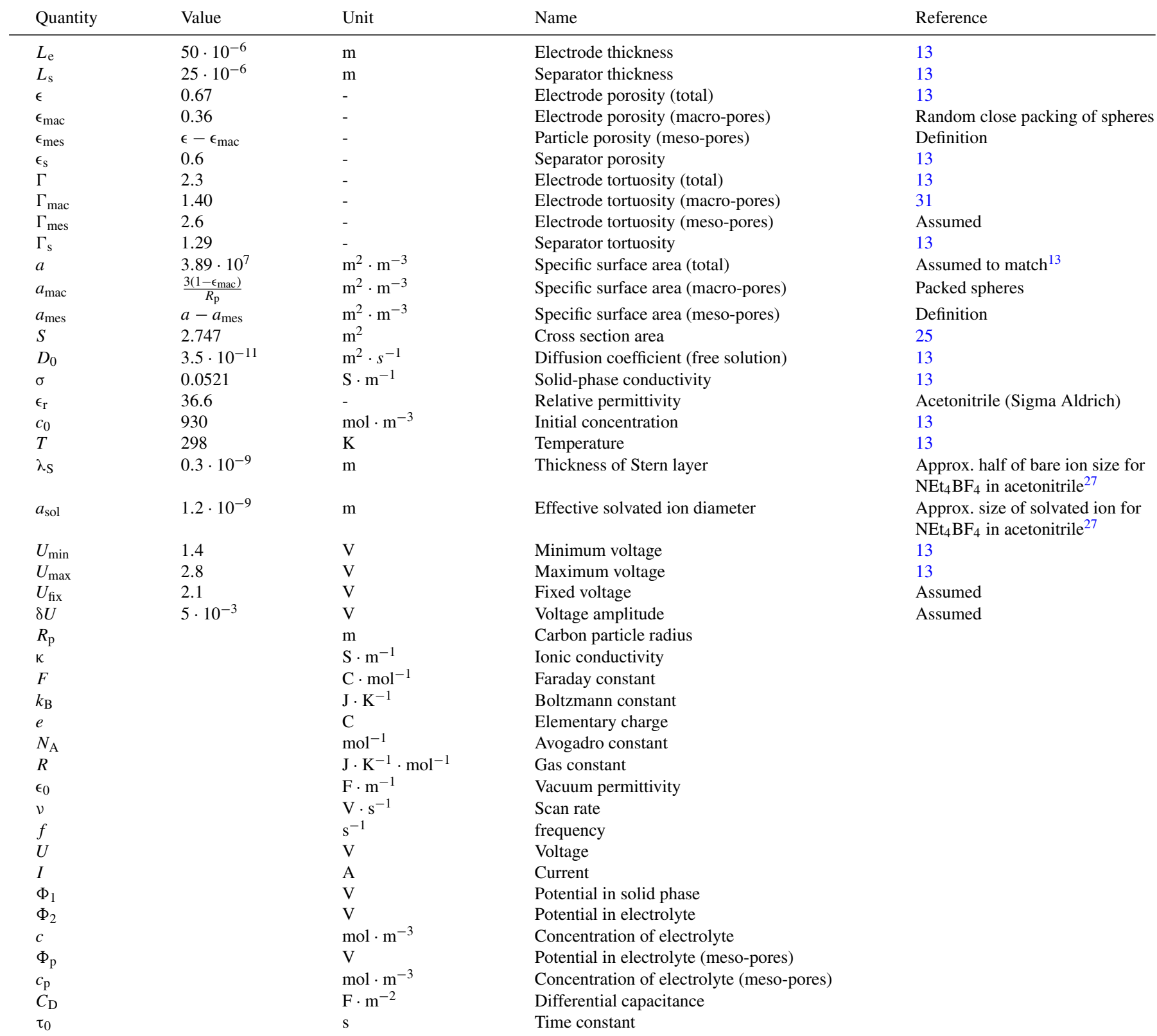




\section{References}

1. B. Wu, M. A. Parkes, V. Yufit, L. De Benedetti, S. Veismann, C. Wirsching, F. Vesper, R. F. Martinez-Botas, A. J. Marquis, G. J. Offer, and N. P. Brandon, International Journal of Hydrogen Energy, 39, 7885 (2014).

2. P. Simon and Y. Gogotsi, Nature Materials, 7, 845 (2008).

3. S. L. Zhang and N. Pan, Advanced Energy Materials, 5, 19 (2015).

4. E. Lust, A. Janes, and M. Arulepp, J. Solid State Electrochem., 8, 488 (2004)

5. S. Yoon, J. H. Jang, B. H. Ka, and S. M. Oh, Electrochimica Acta, 50, 2255 (2005).

6. A. G. Pandolfo, G. J. Wilson, T. D. Huynh, and A. F. Hollenkamp, Fuel Cells, 10, $856(2010)$

7. E. Lust, A. Janes, T. Parn, and P. Nigu, J. Solid State Electrochem., 8, 224 (2004).

8. E. Lust, A. Janes, and M. Arulepp, Journal of Electroanalytical Chemistry, 562, 33 (2004).

9. K. Tonurist, T. Thomberg, A. Janes, T. Romann, V. Sammelselg, and E. Lust, Journal of Electroanalytical Chemistry, 689, 8 (2013).

10. K. Tonurist, A. Jaenes, T. Thomberg, H. Kurig, and E. Lust, Journal of the Electrochemical Society, 156, A334 (2009).

11. I. S. Ike, I. Sigalas, S. Iyuke, and K. I. Ozoemena, Journal of Power Sources, 273, 264 (2015).

12. L. Pilon, H. N. Wang, and A. d'Entremont, Journal of the Electrochemical Society, 162, A5158 (2015)

13. M. W. Verbrugge and P. Liu, Journal of the Electrochemical Society, 152, D79 (2005).

14. S. Allu, B. V. Asokan, W. A. Shelton, B. Philip, and S. Pannala, Journal of Power Sources, 256, 369 (2014).

15. V. Srinivasan and J. W. Weidner, Journal of the Electrochemical Society, 146, 1650 (1999).
16. R. de Levie, Electrochimica Acta, 9, 1231 (1964)

17. M. Eikerling, A. A. Kornyshev, and E. Lust, Journal of the Electrochemical Society, 152, E24 (2005)

18. P. M. Biesheuvel and M. Z. Bazant, Physical Review E, 81, 12 (2010).

19. P. M. Biesheuvel, S. Porada, M. Levi, and M. Z. Bazant, J. Solid State Electrochem., 18, 1365 (2014).

20. T. Kim, J. E. Dykstra, S. Porada, A. van der Wal, J. Yoon, and P. M. Biesheuvel, J. Colloid Interface Sci., 446, 317 (2015).

21. P. M. Biesheuvel, Y. Fu, and M. Z. Bazant, Russ. J. Electrochem., 48, 580 (2012).

22. R. Zhao, M. van Soestbergen, H. H. M. Rijnaarts, A. van der Wal, M. Z. Bazant, and P. M. Biesheuvel, J. Colloid Interface Sci., 384, 38 (2012).

23. J. E. Dykstra, R. Zhao, P. M. Biesheuvel, and A. van der Wal, Water Res., 88, 358 (2016).

24. M. Z. Bazant, M. S. Kilic, B. D. Storey, and A. Ajdari, Advances in Colloid and Interface Science, 152, 48 (2009).

25. R. Drummond, D. A. Howey, and S. R. Duncan, Journal of Power Sources, 277, 317 (2015).

26. F. Beguin and E. Frackowiak, Supercapacitors: Materials, Systems and Applications, Wiley-VCH (2013).

27. R. Lin, P. L. Taberna, J. Chmiola, D. Guay, Y. Gogotsi, and P. Simon, Journal of the Electrochemical Society, 156, A7 (2009).

28. H. N. Wang and L. Pilon, Electrochimica Acta, 64, 130 (2012).

29. P. L. Taberna, P. Simon, and J. F. Fauvarque, Journal of the Electrochemical Society, 150, A292 (2003)

30. A. Janes, H. Kurig, and E. Lust, Carbon, 45, 1226 (2007).

31. P. Y. Lanfrey, Z. V. Kuzeljevic, and M. P. Dudukovic, Chemical Engineering Science, 65, $1891(2010)$ 